\title{
Cognitive impairment and development of dementia in very late-onset schizophrenia-like psychosis: a systematic review
}

\author{
Victoria X Yang ${ }^{1}$ (1), Chun Chiang Sin Fai Lam ${ }^{2}$ and Joseph P M Kane \\ ${ }^{1}$ Medical Student, King's College London GKT School of Medicine, Hodgkin Building, Newcomen St, London, SE1 1UL United Kingdom \\ ${ }^{2}$ Consultant Liaison Psychiatrist, South London and Maudsley NHS Foundation Trust, Department of Psychological Medicine, King's College Hospital, King's \\ College Hospital, Denmark Hill, London, SE5 9RS United Kingdom \\ ${ }^{3}$ Specialist Trainee in Psychiatry of Old Age and General Adult Psychiatry and Academic Clinical Lecturer, Centre for Public Health, Queen's University Belfast, \\ Institute of Clinical Science, Block A, Royal Victoria Hospital, Belfast, BT12 6BA United Kingdom
}

Objectives: This study aimed to review the evidence base regarding cognitive impairment and the development of dementia in patients with very late-onset schizophrenia-like psychosis (VLOSLP).

Methods: We conducted a systematic literature search of PubMed, PsycINFO and Web of Science according to Preferred Reporting Items for Systematic Reviews (PRISMA) guidelines. Two reviewers independently screened records first by title and abstract and then by full text, resolving differences after each stage. Selected studies were assessed for quality using the GRADE system, and data on study design, participants, cognitive ability and rates of developing dementia were extracted and synthesised.

Results: Seventeen publications were identified for review. They were generally poor in quality and heterogenous in design. VLOSLP patients were found to have impaired global cognition compared to non-psychotic controls, but no difference was found between VLOSLP patients and aged early-onset schizophrenia (EOS) patients. No single cognitive domain was consistently affected. Patients with VLOSLP demonstrated significantly higher rates of dementia diagnosis (ranging from $4.4 \%$ over 3 years to $44.4 \%$ over 15 years) than controls, but no difference was found between VLOSLP patients and aged EOS patients.

Conclusions: VLOSLP may not necessarily predict cognitive decline, but few studies have adequately investigated cohorts on a longitudinal basis. Heterogeneity between and within cohorts and varying selection criteria compromise the clinical generalisability of studies investigating the association between VLOSLP and neurodegenerative disease. Further studies on the clinical presentation, cognitive profile and neuropathology of VLOSLP with comparison to EOS/late-onset schizophrenia (LOS) and neurodegenerative disease are needed to better inform the diagnosis and management of VLOSLP.

Received 04 April 2020; Revised 15 April 2021; Accepted 27 May 2021

Key words: Geriatrics, epidemiology, psychosis, schizophrenia, dementia.

\section{Introduction}

Although schizophrenia commonly presents in late adolescent or early adulthood, a small proportion of patients first present with symptoms later in life (Howard et al. 2000). An international consensus defined very late-onset schizophrenia-like psychosis (VLOSLP) as an onset of psychotic symptoms at the age of 60 years or above, which cannot be attributed to a primary affective disorder or structural brain abnormalities (Howard et al. 2000). VLOSLP typically presents with multimodal hallucinations, partition and paranoid delusions (Hanssen et al. 2015) in the absence of formal thought disorder and negative

*Address for correspondence: Dr Joseph PM Kane, Specialist Trainee in Psychiatry of Old Age and General Adult Psychiatry and Academic Clinical Lecturer, Centre for Public Health, Queen's University Belfast, Institute of Clinical Science, Block A, Royal Victoria Hospital, Belfast, BT12 6BA, United Kingdom. Telephone: 02890976350. (Email: joseph.kane@qub.ac.uk) symptoms seen in early-onset schizophrenia (EOS). A two-stage model of onset has been proposed, characterised by initial suspicion, irritability and ideas of reference, followed by florid hallucinations and delusions (Zarit \& Zarit, 2012); however, disease onset remains challenging to pinpoint.

The aetiology of VLOSLP is unclear. VLOSLP differs significantly from EOS in clinical presentation, and unlike EOS, occurs more commonly in women and is less associated with family history or childhood trauma (Reeves \& Brister, 2008). The presentation of VLOSLP also diverges from that of late-onset schizophrenia (LOS), defined as a primary onset of psychotic symptoms at the age of 40 years or above, being associated with a stronger female predominance, brain abnormalities on imaging and overall neuropsychological decline. Compared to VLOSLP, LOS has also been observed to be more commonly associated with positive family history and presenting with negative symptoms (Palmer et al. 2001). 
As VLOSLP often shares clinical features seen in neurodegenerative illnesses, such as cognitive impairment (Palmer et al. 2001), late-life presentation, and behavioural and psychological symptoms (Savva et al. 2009), it has been suggested as a prodromal phase of neurodegenerative dementias (Kørner et al. 2009; Sin Fai Lam et al. 2016; Van Assche et al. 2017). Patients with VLOSLP are subject to greater risk of a dementia than aged-matched controls (Kørner et al. 2009), but the condition does not always predict cognitive or functional decline (Sin Fai Lam et al. 2016). Where impairment does arise, patients with VLOSLP often demonstrate non-progressive impairment similar to static encephalopathy in EOS (Van Assche et al. 2017). The condition is also more prevalent in ethnic minority groups, which may implicate psychosocial stressors as a risk factor (Mitter et al. 2005). Nevertheless, the current consensus is that all cases satisfying diagnostic criteria for schizophrenia, regardless of onset age, fall under the same illness category (Howard et al. 2000).

VLOSLP patients experience substantially incapacitating symptoms and represent a highly vulnerable group. Nevertheless, few systematic reviews have been conducted on the topic, focusing on clinical presentation, neuropsychology and neurobiology (Van Assche et al. 2017; Suen et al. 2019) in LOS and VLOSLP, all of which jointly evaluate outcomes of both conditions. There is debate around whether LOS is driven by the neurodevelopmental origins of EOS or the suggested neurodegenerative processes of VLOSLP (Palmer et al. 2001), and the clinical difference between both conditions remains unclear despite international consensus establishing separate cut-offs for onset age for both conditions (Howard et al. 2000). Consequently, this review focuses on examining only VLOSLP in relation to neurodegenerative disease and cognition.

The objectives of this review are therefore to review cognitive profiles and longitudinal course of cognitive function in patients with VLOSLP and to assess the quality of current evidence.

\section{Methods}

A systematic literature search was conducted based on Preferred Reporting Items for Systematic Reviews (PRISMA) guidelines (Moher et al. 2009) on 5 February 2021 covering PubMed, PsycINFO and Web of Science, using a free text search strategy based on synonyms for 'geriatrics', 'epidemiology', 'psychosis' and 'dementia'. As a consensus on the criteria for VLOSLP was only introduced in 2000 (Howard, 2000), literature predating this uses a range of nomenclature to classify psychosis of first onset after the age of 60 years. Conditions with concepts overlapping with VLOSLP including paraphrenia, affective psychoses and very late-onset delusional disorder (VLODD) were therefore included in search terms for comprehensiveness. Additional studies were identified through searching reference lists of included sources. Only peerreviewed original research available in English and as full texts were withheld for review. Full search terms are described in Supplementary Table 1.

Included studies investigated cognitive functioning, or dementia diagnosis, in patients with a primary onset of psychotic symptoms aged 60 years or above (Howard, 2000) on a cross-sectional or longitudinal basis. We excluded studies investigating patients with pre-VLOSLP history of neurological disease, studies which did not separately describe outcomes of LOS and VLOSLP patient groups, studies which did not specify the age of onset of psychotic symptoms and cross-sectional studies without a control group.

Two-stage screening was performed to determine article eligibility, first by title and abstract and then by full text. Two authors (VY and SFL) independently reviewed titles and abstracts yielded from the systematic search to select full texts for review, and differences were resolved through discussion and mutual agreement. Both authors then independently screened selected full texts against inclusion and exclusion criteria, compared and resolved differences to determine final eligibility. A flowchart of the screening process is displayed in Fig. 1.

Study quality was assessed according to GRADE criteria (Ryan \& Hill, 2016). Due to heterogeneity in study design and outcome measures used, data were synthesised in a narrative structure. Information on study design, participant age and setting, outcome measures and findings was extracted and summarised in Tables 1 and 2. Duration of follow-up, defined as the length of time after which participants were re-assessed for dementia or cognitive ability, was also noted for longitudinal studies.

\section{Results}

\section{Summary of included studies}

\section{Study characteristics}

Nine hundred and sixteen records (excluding duplicates) were identified through the initial search, and 41 records were selected through title and abstract screening. Seventeen studies were identified through full-text review, of which seven were cross-sectional and ten longitudinal. Of seven cross-sectional studies, four compared cognition in VLOSLP patients with that of healthy aged controls (Naguib \& Levy, 1987; Henderson et al. 1998; Moore et al. 2006; Bentall et al. 2009) and two compared VLOSLP patients to young 


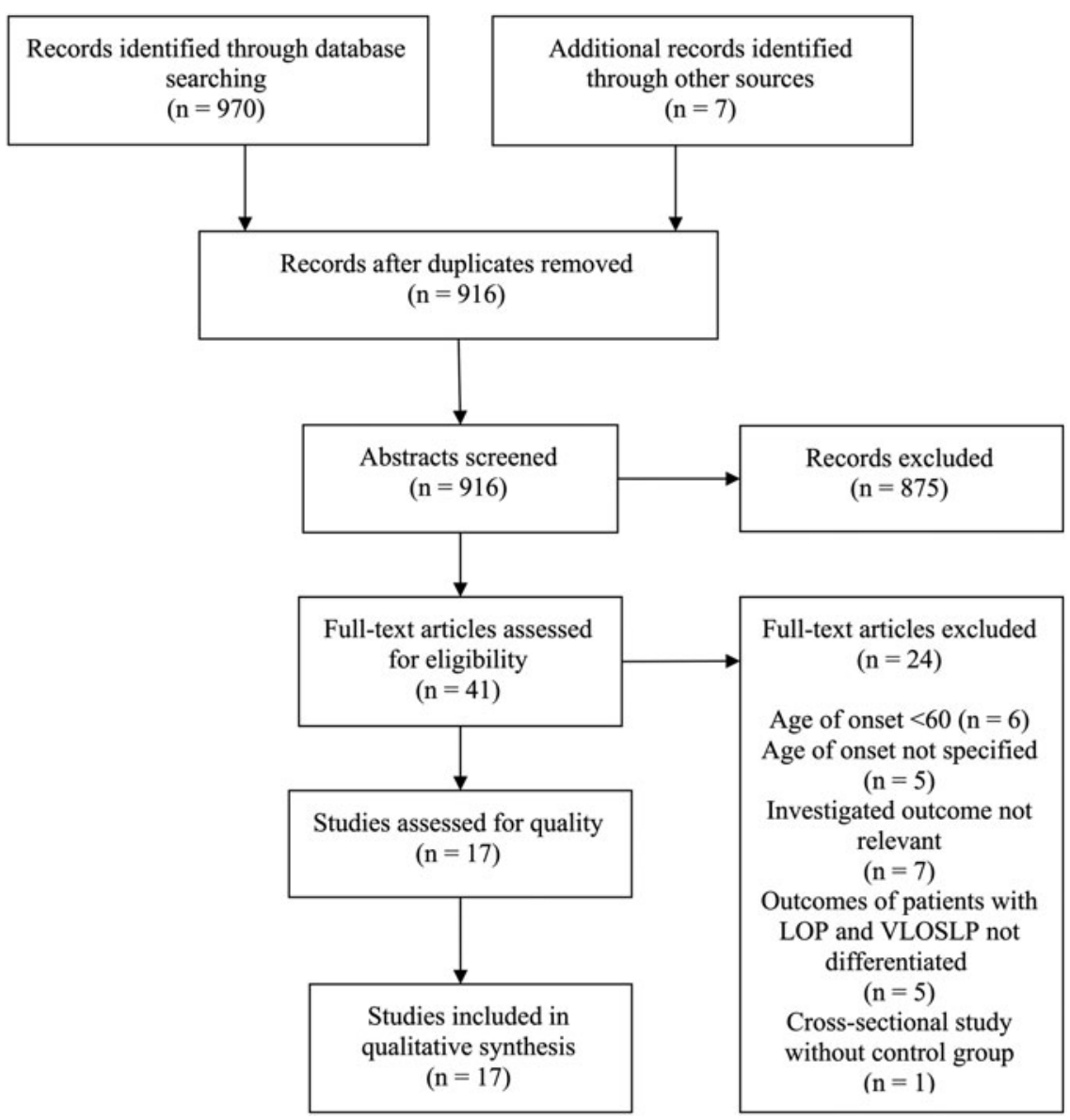

Fig. 1. PRISMA flowchart of study selection process.

patients with EOP (Rodriguez-Ferrera et al. 2004, Hanssen et al. 2015) using a range of assessment tools. One study (Van Assche et al. 2019) compared individual cognitive domains between patients with VLOSLP and patients with neurodegenerative disease, matched for age, years of formal education and general cognitive function assessed using the Mini Mental State Examination (MMSE). Participants came from settings including nursing homes, inpatient and outpatient populations.

Of ten longitudinal studies, six investigated rates of developing dementia over a follow-up period in VLOSLP patients through searching hospital records (Kørner et al. 2008, 2009; Almeida et al. 2019; Kodesh et al. 2020) or assessing patients using diagnostic criteria (Holden, 1987; Östling et al. 2007). Of these, two compared VLOSLP patients to age-matched controls without VLOSLP (Östling et al. 2007; Kodesh et al. 2020), two compared VLOSLP patients to osteoarthritis patients (Kørner et al. 2008, 2009), one compared VLOSLP patients to non-psychotic elderly (Almeida et al. 2019) and one did not use a control group (Holden, 1987). Three studies investigated the course of cognition over a follow-up period using cognitive assessment tools
(Hymas et al. 1989; Howard et al. 1995) or carer impressions (Mazeh et al. 2005), and one study compared rates of mortality from dementia in VLOSLP patients with that of EOS/LOS patients (Talaslahti et al. 2015).

\section{Study quality}

Of 60 outcomes, 45 were rated 'very low' in quality, 13 'low', one 'moderate' and one 'high'. As all included studies were observational in design, all outcomes were assigned a 'low' baseline rating and then downgraded or upgraded based on assessment criteria for risk of bias, inconsistency, indirectness, imprecision, publication bias, large magnitude of effect, dose-response phenomena or the potential of plausible confounding factors to reduce an observed effect. Only studies which were not downgraded for a quality concern were eligible for upgrading (Ryan \& Hill, 2016). Full results of quality assessment are included in Supplementary Table 2.

\section{Global cognitive profiles}

Cognitive ability in patients with VLOSLP was impaired compared to healthy age-matched controls in three studies (Naguib \& Levy, 1987; Moore et al. 
Table 1. Summary of results of cross-sectional studies

\begin{tabular}{|c|c|c|c|c|}
\hline \multicolumn{5}{|l|}{ Cross-sectional studies } \\
\hline Study & Participants & Age (years) & Outcome Measures & Findings \\
\hline Bentall et al. (2009). & $\begin{array}{l}\text { Inpatients and outpatients } \\
\text { VLOSLP }(n=27) \text { andVLODD } \\
\quad(n=2) \\
\text { Healthy aged controls }(n=31)\end{array}$ & $\begin{array}{l}\text { During study } \\
76.90(\mathrm{SD} \pm 5.99) \\
\text { At disease onset } \\
\text { M: } 72.38 \\
\text { SD: } 6.87 \\
\text { During study } \\
\text { M: } 75.6 \\
\text { SD: } 5.54\end{array}$ & WASI, DS Bw, BiaJ, ToMD & $\begin{array}{l}\text { VLOSLP/VLODD patients scored significantly lower on a } \\
\text { multidimensional model of cognitive performance analysed } \\
\text { using the WASI IQ, DS Bw, BiaJ and ToMD tests compared to } \\
\text { healthy aged controls. }\end{array}$ \\
\hline Hanssen et al. (2015). & $\begin{array}{l}\text { Inpatients and outpatients } \\
\text { VLOSLP }(n=28) \\
\text { Healthy controls }(n=290) \\
\text { LOP }(n=24) \\
\text { EOP }(n=24)\end{array}$ & $\begin{array}{l}\text { During study } \\
\text { M: } 75.68 \\
\text { Range: } 72.56-78.80 \\
\text { At onset } \\
\text { M: } 72.39 \\
\text { SD: } 7.72 \\
\text { During study } \\
\text { M:37.55 } \\
\text { Range: } 35.50-39.59 \\
\text { During study } \\
\text { M:58.13 } \\
\text { Range: } 53.02-63.23 \\
\text { At onset } \\
\text { M: } 49.13 \\
\text { SD: } 5.54 \\
\text { During study } \\
\text { M:27.3 } \\
\text { Range: } 26.47-28.24 \\
\text { At onset } \\
\text { M: } 22.19 \\
\text { SD: } 6.49\end{array}$ & $\begin{array}{l}\text { CAMCOG, GWLT, CPT, RST, } \\
\text { abbreviated WAIS-III, } \\
\text { RSPM }\end{array}$ & $\begin{array}{l}\text { Patients with VLOSLP performed marginally better on the } \\
\text { CAMCOG when adjusted for sex, education level, negative } \\
\text { symptoms and remission state }(\mathrm{p}=0.020) \text {. VLOSLP patients } \\
\text { also performed better on the RST compared to LOS patients. } \\
\text { There were no significant differences in performance between } \\
\text { VLOP, LOP and EOP on the WAIS/Raven IQ, GWLT, or CPT } \\
\text { reaction time. VLOP patients had impaired CPT accuracy } \\
\text { scores compared to LOP }(\mathrm{p}=0.047) \text { and EOP patients. }\end{array}$ \\
\hline Henderson et al. (1998). & $\begin{array}{l}\text { Nursing homes } \\
\text { VLOSLP }(n=40) \\
\text { Healthy aged controls }(n=771)\end{array}$ & $\begin{array}{l}\text { During study } \\
\text { M: } 78.9 \\
\text { SD: } 6.5 \\
\text { During study } \\
\text { M: } 76.7 \\
\text { SD: } 5.1\end{array}$ & MMSE, SLMT, EMT, NART & $\begin{array}{l}\text { MMSE, EMT and NART scores were not significantly different } \\
\text { in psychotic and non-psychotic elderly. SLMT scores were sig- } \\
\text { nificantly higher in non-psychotic elderly, } \mathrm{p}=0.001 \text {. }\end{array}$ \\
\hline
\end{tabular}


Table 1. (Continued)

\begin{tabular}{|c|c|c|c|c|}
\hline \multicolumn{5}{|l|}{ Cross-sectional studies } \\
\hline Study & Participants & Age (years) & Outcome Measures & Findings \\
\hline Moore et al. (2006). & $\begin{array}{l}\text { Inpatients and outpatients } \\
\text { VLOSLP }(n=29) \\
\text { Healthy aged controls }(n=30)\end{array}$ & $\begin{array}{l}\text { During study } \\
\text { M:76.90 } \\
\text { SD: } 5.99 \\
\text { During study } \\
\text { M:75.73 } \\
\text { SD: } 5.59\end{array}$ & $\begin{array}{l}\text { WASI IQ, DS Bw, mentalising } \\
\text { task (Snowden et al., } \\
\text { unpublished), BiaJ }\end{array}$ & $\begin{array}{l}\text { VLOSLP patients performed significantly worse on the WASI } \\
\text { IQ test }(\mathrm{p}=0.01) \text {, mentalising task (deception) }(p<0.01) \text { and } \\
\text { digit span backwards test }(p<0.01) \text {. } \\
\text { There was no significant difference in performance between } \\
\text { VLOSLP patients and controls on mentalising task (false } \\
\text { belief) and the beads in a jar test. }\end{array}$ \\
\hline Naguib \& Levy (1987). & $\begin{array}{l}\text { Inpatients and outpatients } \\
\text { Late paraphrenia }(n=43) \\
\text { Healthy aged controls }(n=40)\end{array}$ & $\begin{array}{l}\text { During study } \\
\text { M:75.27 } \\
\text { SD: } 6.29 \\
\text { During study } \\
\text { M:75.85 } \\
\text { SD: } 8.64\end{array}$ & MTS, DCT, DSST & $\begin{array}{l}\text { Patients with paraphrenia performed worse on the MTS } \\
\quad(p<0.001) \text { and DCT }(p<0.002) \text { compared to controls. } \\
\text { There was no significant difference in performance on the DSST. }\end{array}$ \\
\hline $\begin{array}{l}\text { Rodriguez-Ferrera et al. } \\
\text { (2004). }\end{array}$ & $\begin{array}{l}\text { Inpatients and outpatients } \\
\text { VLOP }(n=26) \\
\text { EOP }(n=46)\end{array}$ & $\begin{array}{l}\text { During study } \\
\text { M: } 72 \\
\text { SD: } 7.16 \\
\text { During study } \\
\text { M: } 36 \\
\text { SD: } 11.3\end{array}$ & MMSE, TDRS & $\begin{array}{l}\text { VLOP patients had significantly lower TDRS scores compared } \\
\text { to early onset psychosis (EOP) patients, } \mathrm{p}=0.003 \text {. } \\
\text { No significant difference in MMSE performance was found } \\
\text { between VLOP and EOP patients. }\end{array}$ \\
\hline Van Assche et al. (2019). & $\begin{array}{l}\text { Inpatients and outpatients } \\
\text { VLOSLP }(\mathrm{n}=57)(77.2 \% \mathrm{~F}, \\
22.8 \% \mathrm{M}) \\
\text { DLB }(\mathrm{n}=49)(32.7 \% \mathrm{~F}, 67.3 \% \\
\mathrm{M}) \\
\text { AD }+\mathrm{P}(\mathrm{n}=35)(62.9 \% \mathrm{~F} \\
\quad 37.1 \% \mathrm{M})\end{array}$ & $\begin{array}{l}\text { During study } \\
\text { M: } 79.25 \\
\text { SD: } 7.484 \\
\text { During study } \\
\text { M: } 76.20 \\
\text { SD: } 6.955 \\
\text { During study } \\
\text { M: } 78.80 \\
\text { SD: } 6.286\end{array}$ & $\begin{array}{l}\text { MMSE, Stroop, DS Fw, DS } \\
\text { Bw, RAVLT, BNT, Letter } \\
\text { VF, AVF, VOSP, COTESS }\end{array}$ & $\begin{array}{l}\text { VLOSLP patients had significantly higher scores on the RAVLT } \\
\text { compared to the DLB group ( } \mathrm{p} 0.001) \text { and the AD }+\mathrm{P} \text { group } \\
(p<0.001) \text {. } \\
\text { VLOSLP performed significantly better than the DLB group on } \\
\text { the COTESS house task ( } \mathrm{p} 0.030) \text { and house copy task ( } \mathrm{p} \\
\text { 0.001). } \\
\text { No significant difference in performance on the Stroop, DS Fw } \\
\text { and Bw, BNT, AVF and VOSP was found between VLOSLP, } \\
\text { DLB and AD }+\mathrm{P} \text { patients. }\end{array}$ \\
\hline
\end{tabular}


2006; Bentall et al. 2009). One study found impairment in attention, perceptual speed, motor speed and visual scanning among patients with VLOSLP when compared to age-matched controls (Henderson et al. 1998).

One study found that levels of cognitive impairment in VLOSLP patients were not significantly different from that of aged patients with EOS using the MMSE (Rodriguez-Ferrera et al. 2004). Another study observed better performance from VLOSLP patients on the Cambridge Cognitive Assessment Battery (CAMCOG) compared to LOS patients (Hanssen et al. 2015).

\section{Impairment in individual cognitive domains}

Patients with VLOSLP had impaired reasoning and perceptual organisation ability compared to healthy controls (Östling et al. 2007), and impaired language ability compared to age-matched controls (Bentall et al. 2009; Harris et al. 2014). Memory and recall were also impaired in VLOSLP patients compared to healthy age-matched controls (Naguib \& Levy, 1987). However, VLOSLP patients performed significantly better on short-term auditory-verbal memory and learning tasks compared to Alzheimer's dementia with psychosis and Lewy body dementia patients with similar MMSE scores (Van Assche et al. 2019).

VLOSLP patients had lower accuracy of sustained attention than EOP or LOP patients, although no difference in reaction time of sustained attention measured using the continuous performance test (Hanssen et al. 2015). Attention and vigilance were impaired in patients with VLOSLP compared to healthy controls (Hanssen et al. 2015). However, notably, VLOSLP patients showed significantly less accurate responses on a vigilance task compared with LOS (Hanssen et al. 2015).

Two studies reported that patients with VLOSLP had significant deficits in working memory (Harris et al. 2014; Mazeh et al. 2005) compared to healthy controls.

One study, comparing patients with VLOSLP to those with EOS and LOS, found patients with VLOSLP performed better on response-switching tasks (Hanssen et al. 2015). VLOSLP patients were also found to perform better on tasks assessing visuo-constructive skills compared to patients with Alzheimer's dementia with psychosis and Lewy body dementia; however, no difference was found with visuospatial perception (Van Assche et al. 2019).

\section{Longitudinal course of cognition}

Six studies reported that VLOSLP groups exhibited a significantly greater decline in cognitive function compared to controls over follow-up periods ranging from 6 months to 17.7 years (Hymas et al. 1989; Östling et al. 2007; Kørner et al. 2008, 2009; Almeida et al. 2019; Kodesh et al. 2020). In two studies, follow-up periods were significantly different between subjects and controls (Kørner et al. 2008, 2009).

The proportion of patients with VLOSLP developing dementia ranged from $4.4 \%$ over 3 years (Kørner et al. 2009) to $44.4 \%$ over 15 years (Östling et al. 2007). One study reported that hallucinations conferred additional risk; $60 \%$ of patients experiencing hallucinations developed dementia, compared with $30 \%$ of those with delusions alone (Östling et al. 2007).

When compared with age-matched patients with EOS, VLOSLP participants had a similar rate of dementia but showed greater stability in cognitive and everyday functioning scores (Mazeh et al. 2005).

Two studies found that VLODD groups exhibited a higher rate of developing dementia than age-matched patients with osteoarthritis (Kørner et al. 2008, 2009); one noted that $15 \%$ of VLODD patients compared to $2 \%$ of controls developed dementia within 6 months of initial presentation (Kørner et al. 2008).

One study, in which 13 of 37 patients with paraphrenia were diagnosed with dementia, reported that a higher proportion of patients with lower cognitive scores at initial presentation subsequently developed dementia (Holden, 1987).

\section{Summary}

VLOSLP patients were found to have impaired global cognition compared to age-matched non-psychotic controls, but no strong evidence was found for a difference between VLOSLP patients and aged EOS patients.

Compared to age-matched healthy controls, VLOSLP patients had impairments in reasoning, perception, language, memory and recall, and attention. Compared to age-matched patients with neurodegenerative disease, VLOSLP patients had less impairment of memory, learning and visuo-constructive skills. Compared to agematched patients with EOS/LOS, VLOSLP patients had lower vigilance and accuracy of attention, but performed better on response-switching tasks.

VLOSLP patients generally exhibited a greater decline in cognitive function and higher rates of later developing dementia compared to age-matched controls, but no difference in rate of developing dementia was found between VLOSLP patients and aged EOS patients.

\section{Discussion}

\section{Global cognitive function and individual cognitive domains}

Review of studies reporting on cognitive function in VLOSLP was complicated by heterogeneity of both study populations and outcome measures. Cognitive impairment in patients with VLOSLP compared to 
Table 2. Summary of results of longitudinal Studies.

\begin{tabular}{|c|c|c|c|c|c|}
\hline Study & Participants & Duration of follow-up & Age (years) & Outcome measures & Findings \\
\hline Almeida et al. (2019) & $\begin{array}{l}\text { Treatment context not stated } \\
\text { VLOSLP (sample size not } \\
\text { reported) (men) } \\
\text { Psychosis onset <65 years (sam- } \\
\text { ple size not reported) (men) } \\
\text { Age-matched controls without } \\
\text { psychosis at baseline (n } \\
=37364 \text { ) (men) }\end{array}$ & 17.7 years & $\begin{array}{l}\text { At baseline assessment } \\
65-85\end{array}$ & $\begin{array}{l}\text { WADLS } \\
\text { Dementia from any } \\
\text { cause, as coded by } \\
\text { the International } \\
\text { Classification of } \\
\text { Diseases } 9 \text { \& 10, was } \\
\text { the primary outcome } \\
\text { of interest. }\end{array}$ & $\begin{array}{l}\text { At follow-up, VLOSLP patients were found } \\
\text { to have a significantly higher rate of } \\
\text { dementia compared to patients without a } \\
\text { psychotic disorder at baseline. } \\
\text { No significant difference was found } \\
\text { between VLOSLP patients and age- } \\
\text { matched EOS/LOS patients. }\end{array}$ \\
\hline Holden, (1987). & $\begin{array}{l}\text { Hospitalised at baseline } \\
\text { assessment } \\
\text { VLOP }(\mathrm{n}=37)\end{array}$ & 10 years & $\begin{array}{l}\text { Did not progress to dementia } \\
\text { M: } 68.5 \\
\text { Range: } 60-79 \\
\text { Progressed to dementia } \\
\text { M: } 72.5 \\
\text { Range: } 60-82\end{array}$ & GWQ & $\begin{array}{l}\text { At follow-up after } 10 \text { years, } 13 \text { (35\%) cases } \\
\text { were diagnosed as 'organic psychosis' } \\
\text { which progressed to dementia; } 65 \% \text { were } \\
\text { not diagnosed with dementia. Patients } \\
\text { who progressed to dementia had signifi- } \\
\text { cantly lower GWQ scores at baseline } \\
\text { assessment. }\end{array}$ \\
\hline Howard et al. (1995). & $\begin{array}{l}\text { Inpatients and outpatients } \\
\operatorname{VLOSLP}(n=23)(20 \mathrm{~F}, 3 \mathrm{M})\end{array}$ & 2 years & M: 79.91 & MMSE & $\begin{array}{l}\text { At baseline assessment, all patients scored } \\
\text { above } 24 \text { on the MMSE. } \\
\text { At follow-up assessment, } 3 \text { of } 23(13 \%) \\
\text { patients scored below } 24 \text { on the MMSE. }\end{array}$ \\
\hline Hymas et al. (1989). & $\begin{array}{l}\text { Inpatients and outpatients } \\
\text { Late paraphrenia }(\mathrm{n}=42) \\
\text { Healthy aged controls at baseline } \\
\quad(\mathrm{n}=40)(31 \mathrm{~F}, 9 \mathrm{M}) \\
\text { Healthy aged controls at follow- } \\
\quad \text { up }(\mathrm{n}=23)(18 \mathrm{~F}, 5 \mathrm{M})\end{array}$ & $\begin{array}{l}\text { M: } 3.7 \text { years } \\
\text { Range: } 2.8-5.3 \\
\text { M: } 3.7 \text { years } \\
\text { Range: } 3.4-4.3\end{array}$ & $\begin{array}{l}\text { Patients at baseline assess- } \\
\text { ment } \\
\text { M: } 75.27 \\
\text { SD: } 6.29 \\
\text { Controls at baseline } \\
\text { M: } 75.9 \\
\text { SD: } 8.6 \\
\text { Controls at follow-up } \\
\text { M: } 77.9 \\
\text { SD: } 9\end{array}$ & MTS & $\begin{array}{l}\text { On both initial assessment and follow-up } \\
\text { assessment, patients with paraphrenia } \\
\text { performed significantly worse on the } \\
\text { MTS than healthy controls. } \\
\text { Both the paraphrenia group }(\mathrm{p}=0.005) \text { and } \\
\text { the control group }(\mathrm{p}=0.025) \text { showed a } \\
\text { significant decline in MTS scores. } \\
\text { Patients with paraphrenia showed a signifi- } \\
\text { cantly greater decline in MTS scores, } \\
\text { with } 14 \text { of } 42 \text { rated as 'cognitively } \\
\text { impaired' }(33 \%) \text { at follow-up. }\end{array}$ \\
\hline Kodesh et al. (2020). & $\begin{array}{l}\text { Treatment context not stated } \\
\text { VLOS }(\mathrm{n}=329)(62.6 \% \mathrm{~F} \text {, } \\
\quad 37.4 \% \mathrm{M}) \\
\text { Age-matched controls }(\mathrm{n}=93791) \\
(53.5 \% \mathrm{~F}, 46.5 \% \mathrm{M})\end{array}$ & 4.8 years & $\begin{array}{l}\text { At baseline } \\
\text { M: } 68.9 \\
\text { SD: } 7.1\end{array}$ & $\begin{array}{l}\text { Meuhedet dementia } \\
\text { registry } \\
\text { Dementia was defined } \\
\text { based on the } \\
\text { International } \\
\text { Classification of } \\
\text { Diseases } 9 \text { \& } 10 .\end{array}$ & $\begin{array}{l}\text { VLOS patients had a significantly higher } \\
\text { rate of developing dementia }(\mathrm{n}=64 \\
19.52 \%) \text { compared to non-VLOS patients } \\
(\mathrm{n}=5962,6.4 \%), p<0.001 \text {. }\end{array}$ \\
\hline
\end{tabular}


Table 2. (Continued)

\begin{tabular}{|c|c|c|c|c|c|}
\hline \multicolumn{6}{|l|}{ Longitudinal studies } \\
\hline Study & Participants & Duration of follow-up & Age (years) & Outcome measures & Findings \\
\hline Kørner et al. (2008). & $\begin{array}{l}\text { Treatment context not stated } \\
\text { VLODD }(\mathrm{n}=1437)(77.5 \% \mathrm{~F}) \\
\text { Controls: } \\
\text { OA: } 7302(63.6 \% \mathrm{~F})\end{array}$ & $\begin{array}{l}\text { Median: } 1.87 \text { years } \\
\text { Quartiles: } 0.69,3.64 \\
\text { Median: } 4.40 \text { years } \\
\text { Quartiles: } 2.28,6.28\end{array}$ & $\begin{array}{l}\text { At first discharge } \\
\text { Median: } 79.4 \\
\text { Quartiles: } 72.6,85.8 \\
\text { At first discharge } \\
\text { Median: } 71.3 \\
\text { Quartiles: } 65.8,76.7\end{array}$ & $\begin{array}{l}\text { DPCR, DNHR } \\
\text { Dementia was defined } \\
\text { as main diagnosis of } \\
\mathrm{AD} / \mathrm{VaD} / \text { unspeci- } \\
\text { fied dementia based } \\
\text { on the International } \\
\text { Classification of } \\
\text { Diseases } 10 .\end{array}$ & $\begin{array}{l}\text { A significantly higher proportion of } \\
\text { patients with VLODD were later diag- } \\
\text { nosed with dementia }(15.2 \% \text { ) compared } \\
\text { to patients with OA }(2.1 \%), p<0.0001 \text {. } \\
\text { VLODD patients had a higher relative risk } \\
\text { (number) of developing dementia in the } \\
\text { first } 6 \text { months after diagnosis compared } \\
\text { to OA patients. Male VLODD patients } \\
\text { had a higher relative risk than women. }\end{array}$ \\
\hline Kørner et al. (2009). & $\begin{array}{l}\text { Treatment context not stated } \\
\text { VLOSLP }(n=409)(64.3 \% \text { F) } \\
\text { OA }(n=7303)(63.6 \% \text { F) }\end{array}$ & $\begin{array}{l}\text { Median: } 3.00 \\
\text { Quartiles: } 1.25,4.78 \\
\text { Median: } 4.41 \\
\text { Quartiles: } \\
2.28,6.29\end{array}$ & $\begin{array}{l}\text { At first discharge } \\
\text { Median: } 71.19 \\
\text { Quartiles: } 64.88,77.89 \\
\text { At first discharge } \\
\text { Median: } 71.19 \\
\text { Quartiles: } 65.78,76.74\end{array}$ & $\begin{array}{l}\text { DPCR, DNHR } \\
\text { Dementia was defined } \\
\text { as main diagnosis of } \\
\mathrm{AD} / \mathrm{VaD} / \text { unspeci- } \\
\text { fied dementia based } \\
\text { on the International } \\
\text { Classification of } \\
\text { Diseases } 10 .\end{array}$ & $\begin{array}{l}\text { VLOSLP patients had a significantly higher } \\
\text { rate of being subsequently diagnosed } \\
\text { with dementia }(4.4 \%) \text { compared to } \\
\text { healthy controls }(2.15 \%), p<0.0001 \text {. }\end{array}$ \\
\hline Mazeh et al. (2005). & $\begin{array}{l}\text { Inpatients and outpatients } \\
\text { Primary caregivers of VLOSLP } \\
\text { patients }(n=21) \text { (patients: } 15 \mathrm{~F} \text {, } \\
6 \mathrm{M}) \\
\text { Inpatients } \\
\text { Primary caregivers of elderly EOS } \\
\text { patients }(\mathrm{n}=21) \text { (patients: } 15 \mathrm{~F} \text {, } \\
6 \mathrm{M})\end{array}$ & 6-30 months & $>70$ & $\begin{array}{l}\text { Telephone interviews } \\
\text { with primary carers }\end{array}$ & $\begin{array}{l}13 \text { of } 16 \text { living VLOSLP patients appeared } \\
\text { cognitively intact at follow-up, } 3(18.8 \%) \\
\text { showed decline. } \\
16 \text { of } 19 \text { living EOS patients appeared cog- } \\
\text { nitively intact at follow-up, } 3(15.8 \%) \\
\text { showed decline. }\end{array}$ \\
\hline Östling et al. (2007). & $\begin{array}{l}\text { Inpatients and outpatients } \\
\text { VLOSLP }(n=18) \\
\text { Age-matched controls }(n=349)\end{array}$ & 15 years & $\begin{array}{l}\text { At baseline assessment } \\
70 \\
\text { At follow-up } \\
85\end{array}$ & $\begin{array}{l}\text { Diagnostic and } \\
\text { Statistical Manual of } \\
\text { Mental Disorders, } \\
\text { 3rd Edition } \\
\text { (Revised) (DSM-III- } \\
\text { R) criteria }\end{array}$ & $\begin{array}{l}\text { A significantly higher proportion of } \\
\text { VLOSLP patients ( } 44.4 \% \text { ) developed } \\
\text { dementia compared to controls }(25.2 \%) \\
p<0.01\end{array}$ \\
\hline Talaslahti et al. (2015). & $\begin{array}{l}\text { Treatment context not stated } \\
\text { VLOSLP }(n=918) \\
\text { EOS/LOS }(n=6142)\end{array}$ & 10 years & $\begin{array}{l}\text { At baseline assessment } \\
>65\end{array}$ & FHDR, NCDRSF & $\begin{array}{l}\text { SMR from dementia was found to be sig- } \\
\text { nificantly higher in VLOSLP patients } \\
\text { than in elderly patients with early- or } \\
\text { late-onset schizophrenia, } p<0.001 \text {. }\end{array}$ \\
\hline
\end{tabular}

WADLS, Western Australia Data Linkage System; VLOP, very late-onset psychosis; GWQ, Gresham Ward Questionnaire; VLOS, Very Late-Onset Schizophrenia; VLODD, Very Late-Onset Delusional Disorder; OA, Osteoarthritis; DPCR, Danish Psychiatric Central Register; DNHR, Danish National Hospital Register; AD, Alzheimer's Disease; VaD, vascular dementia; FDHR, Finnish Hospital Discharge Register; NCDRSF, National Causes of Death Register of Statistics Finland; SMR, Standard Mortality Ratio; VLOSLP, very late-onset schizophrenia-like psychosis; LOS, late-onset schizophrenia; EOS, early-onset schizophrenia; MMSE, Mini Mental State Examination.

An expanded version of this table is included in online-only material as Supplementary Table 4. 
healthy age-matched controls was observed only in three studies (Naguib \& Levy, 1987; Moore et al. 2006; Bentall et al. 2009). Evidence comparing VLOSLP with LOS patients was conflicting, with VLOSLP patients outperforming LOS patients on the CAMCOG in one study (Hanssen et al. 2015) but performing similarly on the MMSE in another (Rodriguez-Ferrera et al. 2004). Small sample sizes in both studies may account for this inconsistency in findings.

No single cognitive domain was observed to be consistently affected in VLOSLP compared to controls. Executive dysfunction, although commonly observed deficits in all forms of schizophrenia (Orellana \& Slachevsky, 2013), did not appear to be consistently impaired. Deficits in attention were however noted among patients with VLSOLP when compared to both healthy controls and individuals with LOS and EOS (Hanssen et al. 2015).

VLOSLP patients were found to have less impaired short-term audio-visual memory and learning and visuo-constructive skills compared to patients with psychotic Alzheimer's dementia and Lewy body dementia with similar MMSE scores, suggesting possible sparing of these domains (Van Assche et al. 2019).

As both attentional and executive function impairments are important clinical features of neurodegenerative conditions, longitudinal assessment of this patient group for later dementia diagnosis could be helpful in defining and separating the clinical profiles of both conditions.

\section{Dementia in patients with VLOSLP}

VLOSLP patient groups exhibited significantly higher rates of dementia diagnosis than non-psychotic controls over follow-up periods ranging from 6 months to 17.7 years (Hymas et al. 1989; Östling et al. 2007; Korner et al. 2008, 2009; Almeida et al. 2019; Kodesh et al. 2020). Rates of dementia diagnosis ranged from $4.4 \%$ over 3 years (Kørner et al. 2009) to $44.4 \%$ over 15 years (Östling et al. 2007), which may be attributable to differences in study design and methods of dementia diagnosis including Gresham Ward Questionnaire (GWQ), International classification of diseases (ICD)-10 and DSM-III-R.

Cohort prevalence of dementia was found to increase with longer follow-up periods as expected. Only one study identified a clinical risk factor for progression to dementia, the presence of hallucinations (Östling et al. 2007). Another reported lower cognitive scores at presentation as a risk factor for later progression (Holden, 1987), but it is unclear if this represented a risk factor for subsequent dementia, or indicate possible misdiagnosis of a prodromal phase of neurodegenerative disease. Kørner and colleagues' striking observation of a marked difference in progression to dementia within 6 months of initial presentation between patients with VLODD may raise the possibility of an initial high-risk period for progression to dementia, which subsequently regresses to a rate more comparable with that of controls (Kørner et al. 2008). However, it is worth noting that rate of progression to dementia differed significantly between two studies conducted by the same group in two consecutive years even with near-identical study design and methods (Kørner et al. 2008, 2009). The study investigating VLODD observed a rate of $15.2 \%$ despite a shorter followup period of 1.87 years (Kørner et al. 2008), while the study invsetigating VLOSLP observed $4.4 \%$ over 3 years (Kørner et al. 2009), contradicting earlier findings of hallucinations as a risk factor for progression (Östling et al. 2007). A possible explanation for this inconsistency could be common misdiagnosis of early-stage dementia for VLODD initially, contributing to a high rate of subsequent diagnosis.

Importantly, irrespective of differences in study design and outcome measures used, less than half of VLOSLP patients developed dementia in all studies, even when $25 \%$ of healthy matched controls did (Östling et al. 2007), indicating a significant patient proportion without cognitive decline and supporting a distinct or diverging pathophysiological pathway from that of neurodegenerative disease. Even where male VLOSLP patients had higher rates of developing dementia compared to non-psychotic controls, there was no significant difference compared to aged male EOS/LOS patients (Almeida et al. 2019), suggesting a similar course of cognition in old age across all schizophrenias regardless of onset age.

One study directly compared patients with VLOSLP to those with psychotic Alzheimer's dementia and Lewy body dementia (Van Assche et al. 2019). In this study, dementia patients were diagnosed using a combination of clinical features, biomarkers and imaging, and VLOSLP patients were deemed to show no evidence of neurologic or major affective disorder. Participants were matched for MMSE scores, with both groups having a mean score just above 24, implying a similar stage of functional decline. Although the process of selecting for MMSE-matched participants was not elaborated on, the absence of neurodegenerative signs characteristic of dementias in the VLOSLP group despite similar levels of cognitive decline could point towards diverging mechanisms in both groups.

\section{Interpretation of findings}

Study quality was generally poor. Using the GRADE system (Ryan \& Hill, 2016), five studies were attributed lower ratings for quality due to imprecision involving the use of meta-analysis to derive significant results 
(Bentall et al. 2009) and wide confidence intervals (Naguib \& Levy, 1987; Hymas et al. 1989; Henderson et al. 1998; Mazeh et al. 2005). Studies rated as lower quality due to risk of bias had a low response rate (Henderson et al. 1998; Östling et al. 2007), excluded VLOSLP patients with clinical dementia for cognitive assessment at followup (Hanssen et al. 2015) or used sample selection methods which could have excluded more EOP patients with more severe illness (Rodriguez-Ferrera et al. 2004). Studies rated down for indirectness relative to our research question investigated mortality from dementia as a primary outcome (Talaslahti et al. 2015) or sampled only male patients (Almeida et al. 2019) despite known female predominance in VLOSLP patients (Howard, 2000). One study was attributed lower ratings for inconsistency as different groups of patients were administered different cognitive tasks without explanation of the selection process (Van Assche et al. 2019). Two studies were rated highly for large sample sizes and risk ratios, implying a large magnitude of effect (Kørner et al. 2008; Kodesh et al. 2020). One study rated highly for plausible confounding factors reducing observed effects, as VLODD patients exhibited higher rates of dementia despite shorter follow-up periods compared to controls (Kørner et al. 2008).

Included studies spanned 33 years and were subject to multiple changes in the concept of the disease and shifting diagnostic criteria. This lack of uniformity in selection criteria implies a lack of generalisability of individual study findings. A wide range of measures were used across all studies including clinical diagnosis, functional decline and cognitive test scores. No two studies employed the same primary outcome measure, and studies examining functional decline and clinical diagnosis of dementia notably did not standardise assessments. Of all studies reporting development of dementia, only one adopted a rating scale routinely used in clinical practice, the MMSE; this, or CAMCOG, was used as an outcome measure in only 4 of 17 included studies.

Three non-retrospective longitudinal studies had high dropout rates (23\%-41\%) (Hymas et al. 1989; Mazeh et al. 2005; Östling et al. 2007). More generally, studies failed to consistently describe disease duration, points at which patients' ages were measured, or specify length of time in changing cognitive function. The heterogeneity of outcome measures used made it difficult to compare studies, especially as some studies utilised subjective measures such as collateral interviews (Mazeh et al. 2005). Studies further exploring this relationship should aim to follow-up participants up for 10 years or longer; only four studies included in this review adopted follow-up periods greater than 5 years (Holden, 1987; Östling et al. 2007; Talaslahti et al. 2015; Almeida et al. 2019).
All studies were limited by an absence of neuropathological data, the presence of which might indicate if VLOSLP represents a risk factor for dementia, a prodromal phase or a different manifestation of shared pathophysiology. One pathological study has associated VLOSLP with ventricular enlargement, generalised atrophy and vascular brain damage (Antonova et al. 2004). A high incidence of Lewy body dementia and argyrophilic grain disease pathology, including deterioration of the frontal and temporal lobes have also been detected in VLOSLP patients (Cloud et al. 1996; Nagao et al. 2014). As Lewy body dementia is also associated with symptoms such as delusions and hallucinations, research comparing VLOSLP with these conditions, perhaps with the aid of neuroimaging or neuropathological data, could be helpful in improving differential diagnosis and understanding pathophysiology.

Our systematic, replicable search strategy, which adopted a fixed framework to analyse study quality, represents the greatest strength of this review. We did not conduct meta-analysis, but study heterogeneity and small sample size precluded this. Nevertheless, the absence of meta-analysis does render the review prone to interpretation bias.

\section{Current and future directions}

This study systematically reviewed 17 studies investigating cognitive impairment in patients with VLOSLP, and the proportion of such patients who develop dementia. Notwithstanding the caveats to interpretation discussed above, our overall findings point towards separate pathophysiology between VLOSLP and neurodegenerative disease and suggest similar courses of cognition between EOS, LOS and VLOSLP in old age.

A major concern our findings raise is the diagnostic integrity of VLOSLP. Despite an international consensus on criteria, the current definition of VLOSLP still encompasses a very broad range of presentations, and this heterogeneity is reflected in the selection of patient samples in included studies. One study comparing neurodegenerative disease to VLOSLP sampled 57 patients with an onset of psychosis after the age of 60 years, of which presentations ranged from mild neurocognitive disorder with schizophrenia spectrum disorder to mild neurocognitive disorder with psychosis, pure schizophrenia, delusional disorder, brief psychotic disorder, adjustment disorder with psychosis and initial anxiety with persisting psychosis (Van Assche et al. 2019). This diversity in presentation calls into question the categorisation of these patients under the same diagnosis, and the validity of comparison to dementia patients in this capacity.

In most studies, VLOSLP patients were selected on the basis of showing no evidence of neurological 
disorder; however, it was not established if imaging or biomarker investigations were conducted during the diagnostic process. Furthermore, as EOS, LOS and VLOSLP are distinguished by cut-offs for onset age, diagnostic delays or missed diagnoses can additionally conflate one condition with another.

While trends in clinical presentation, such as lack of negative symptoms, have been identified, the current lack of specificity in diagnostic guidelines creates uncertainty around the selection of VLOSLP patient samples, compromising the validity of findings even in welldesigned studies. More research is therefore required to characterise VLOSLP as a clinical syndrome to strengthen the diagnostic process and enhance the applicability of subsequent findings.

Longitudinal cohort studies comparing the progression of clinical presentation, cognition and pathology in VLOSLP patients to that of EOS/LOS patients over time are needed to better define VLOSLP as a clinical syndrome and establish more robust selection criteria. Following that, studies comparing VLOSLP to neurodegenerative disease using standardised assessment tools and neuropathological investigations could shed light on any shared or diverging pathophysiology.

As older age groups are the fastest growing demographic in the world, VLOSLP and neurodegenerative conditions may become increasingly prominent in the global healthcare landscape. Managing VLOSLP is challenging as patients typically lack insight (Folsom et al. 2006) and are difficult to engage with (Sin Fai Lam et al. 2016) and often have morbidities and concurrent drug treatments increasing the risk of antipsychotic drug-related morbidity and mortality (Reeves et al. 2018). This difficulty in engagement, amplified by a level of cognitive impairment in this patient cohort, could necessitate more assertive engagement methods, as successfully demonstrated with Early Intervention in Psychosis services for EOS patients (Neale \& Kinnair, 2017). Longitudinal follow-up should also be considered in VLOSLP patients who have lost contact with services, using methods such as linkage to national mortality databases, in order to better establish morbidity and mortality outcomes in disengaged patients.

\section{Acknowledgements}

The authors acknowledge the constructive criticism offered by the peer reviewers; their input unquestionably helped produce a more robust article.

\section{Financial Support}

This research received no specific grant from any funding agency, commercial or not-for-profit sectors.

\section{Conflicts of interest}

The authors have no conflicts of interest to disclose.

\section{Ethical Standards}

The authors assert that all procedures contributing to this work comply with the ethical standards of the relevant national and institutional committee on human experimentation with the Helsinki Declaration of 1975, as revised in 2008.

\section{Author contribution statement}

All authors declare that they have met the ICMJE criteria for authorship.

VY was responsible for the acquisition of data, analysis and interpretation, drafting of article, critical revision and approval of version of manuscript to be published. SFL was responsible for the acquisition of data, critical revision and approval of the manuscript. JK was responsible for the critical revision and approval of the manuscript.

\section{Supplementary material}

To view supplementary material for this article, please visit https:/ / doi.org/10.1017/ipm.2021.48

\section{References}

Almeida OP, Ford AH, Hankey GJ, Yeap BB, Golledge J, Flicker L (2019). Risk of dementia associated with psychotic disorders in later life: the health in men study (HIMS). Psychological Medicine 49, 232-242.

Antonova E, Sharma T, Morris R, Kumari V (2004). The relationship between brain structure and neurocognition in schizophrenia: A selective review. Schizophrenia Research 70, 117-145.

Bentall RP, Rowse G, Shryane N, Kinderman P, Howard R, Blackwood N, Moore S, Corcoran R (2009). The Cognitive and Affective Structure of Paranoid Delusions. Archives of General Psychiatry 66, 236.

Cloud BS, Carew TG, Rothenberg H, Malloy P, Libon DJ (1996). A case of late-onset psychosis: integrating neuropsychological and SPECT data. Journal of Geriatric Psychiatry and Neurology 9, 146-153.

Folsom DP, Lebowitz BD, Lindamer LA, Palmer BW, Patterson TL, Jeste DV (2006). Schizophrenia in late life: emerging issues. Dialogues in Clinical Neuroscience 8, 45-52.

Hanssen M, van der Werf M, Verkaaik M, Arts B, Myin-Germeys I, van Os J, Verhey F, Kohler S (2015). Comparative Study of Clinical and Neuropsychological Characteristics Between Early-, Late and Very-Late-Onset Schizophrenia-Spectrum Disorders. American Journal of Geriatric Psychiatry 23, 852-862. 
Harris BS, Kotsopoulos EJ, Yamin S (2014). Phenotypic cognitive impairment in late-onset delusional disorder. International Psychogeriatrics 26, 965-975.

Henderson AS, Korten AE, Levings C, Jorm AF, Christensen H, Jacomb PA, Rodgers B (1998). Psychotic Symptoms in the Elderly: a Prospective Study in a Population Sample. International Journal of Geriatric Psychiatry 492, 484-492.

Holden NL (1987). Late paraphrenia or the paraphrenias? A descriptive study with a 10-year follow-up. The British Journal of Psychiatry 150, 635-639.

Howard R (2000). Late-Onset Schizophrenia and Very-Late-Onset Schizophrenia-Like Psychosis: an International Consensus. American Journal of Psychiatry 157, 172-178.

Howard R, Dennehey J, Lovestone S, Birkett J, Sham P, Powell J, Castle D, Murray R, Levy R (1995). Apolipoprotein-E Genotype and Late Paraphrenia. International Journal of Geriatric Psychiatry 10, 147-150.

Hymas N, Naguib M, Levy R (1989). Late paraphrenia - a follow-up study. International Journal of Geriatric Psychiatry 4, 23-29.

Kodesh A, Goldberg Y, Rotstein A, Weinstein G, Reichenberg A, Sandin S, Levine SZ (2020). Risk of dementia and death in very-late-onset schizophrenia-like psychosis: A national cohort study. Schizophrenia Research 223, 220-226.

Korner A, Lopez AG, Lauritzen L, Andersen PK, Kessing LV (2008). Delusional disorder in old age and the risk of developing dementia-a nationwide register-based study. Aging \& Mental Health 12, 625-629.

Korner A, Lopez AG, Lauritzen L, Andersen PK, Kessing LV (2009). Late and very-late first-contact schizophrenia and the risk of dementia-a nationwide register based study. International Journal of Geriatric Psychiatry 24, 61-67.

Mazeh D, Zemishlani C, Aizenberg D, Barak Y (2005). Patients With Very-Late-Onset Schizophrenia-like Psychosis: A Follow-Up Study. American Journal Geriatric Psychiatry 13, 417-419.

Mitter P, Reeves S, Romero-Rubiales F, Bell P, Stewart R, Howard R (2005). Migrant status, age, gender and social isolation in very late-onset schizophrenia-like psychosis. International Journal of Geriatric Psychiatry 20, 1046-1051.

Moher D, Liberati A, Tetzlaff J, Altman DG, Group TP (2009). Preferred Reporting Items for Systematic Reviews and Meta-Analyses: the PRISMA Statement. PLoS Medicine 6, e1000097.

Moore R, Blackwood N, Corcoran R, Rowse G, Kinderman P, Bentall R, Howard R (2006). Misunderstanding the intentions of others? An exploratory study of the cognitive etiology of persecutory delusions in very lateonset schizophrenia-like psychosis. American Journal of Geriatric Psychiatry 14, 410-418.

Nagao S, Yokota O, Ikeda C, Takeda N, Ishizu H, Kuroda S, Sudo K, Terada S, Murayama S, Uchitomi Y (2014). Argyrophilic grain disease as a neurodegenerative substrate in late-onset schizophrenia and delusional disorders. European Archives of Psychiatry and Clinical Neuroscience 264, 317-331.

Naguib M, Levy R (1987). Late paraphrenia: Neuropsychological impairment and structural brain abnormalities on computed tomography. International Journal of Geriatric Psychiatry 2, 83-90.

Neale A, Kinnair D (2017). Early intervention in psychosis services. British Journal of General Practice 67, 370-371.

Orellana G, Slachevsky A (2013). Executive Functioning in Schizophrenia. Frontiers in Psychiatry 4, 35.

Östling S, Pálsson SP, Skoog I (2007). The incidence of first-onset psychotic symptoms and paranoid ideation in a representative population sample followed from age 70-90 years. Relation to mortality and later development of dementia. International Journal of Geriatric Psychiatry 22, 520-528.

Palmer BW, McClure FS, Jeste DV (2001). Schizophrenia in late life: findings challenge traditional concepts. Harvard Review of Psychiatry 9, 51-58.

Reeves RR, Brister JC (2008). Psychosis in late life: emerging issues. Journal of Psychosocial Nursing and Mental Health Services 46, 45-52.

Reeves S, Eggleston K, Cort E, McLachlan E, Brownings S, Nair A, Greaves S, Smith A, Dunn J, Marsden P, Kessler R, Taylor D, Bertrand J, Howard R (2018). Therapeutic D2/3 receptor occupancies and response with low amisulpride blood concentrations in very late-onset schizophrenia-like psychosis (VLOSLP). International Journal of Geriatric Psychiatry 33, 396-404.

Rodriguez-Ferrera S, Vassilas CA, Haque S (2004). Older people with schizophrenia: a community study in a rural catchment area. International Journal of Geriatric Psychiatry 19, 1181-1187.

Ryan R, Hill S (2016). How to GRADE the quality of the evidence. Available from: http:/ / cccrg.cochrane.org/ author-resources. Accessed 12 February 2018.

Savva GM, Zaccai J, Matthews FE, Davidson JE, McKeith I, Brayne C (2009). Prevalence, correlates and course of behavioural and psychological symptoms of dementia in the population. The British Journal of Psychiatry 194, 212-219.

Sin Fai Lam CC, Reeves SJ, Stewart R, Howard R (2016). Service and treatment engagement of people with very late-onset schizophrenia-like psychosis. BJPsych Bulletin 40, 185-186.

Suen YN, Wong SMY, Hui CLM, Chan SKW, Lee EHM, Chang WC, Chen EYH (2019). Late-onset psychosis and very-late-onset-schizophrenia-like-psychosis: an updated systematic review. International Review of Psychiatry 31, 523-542.

Talaslahti T, Alanen HM, Hakko H, Isohanni M, Häkkinen U, Leinonen E (2015). Patients with very-lateonset schizophrenia-like psychosis have higher mortality rates than elderly patients with earlier onset schizophrenia. International Journal of Geriatric Psychiatry 30, 453-459.

Van Assche L, Morrens M, Luyten P, Van de Ven L, Vandenbulcke M (2017). The neuropsychology 
and neurobiology of late-onset schizophrenia and very-late-onset schizophrenia-like psychosis: A critical review. Neuroscience \& Biobehavioral Reviews 83, 604-621.

Van Assche L, Van Aubel E, Van de Ven L, Bouckaert F, Luyten P, Vandenbulcke M (2019). The

Neuropsychological Profile and Phenomenology of Late Onset Psychosis: A Cross-sectional Study on the
Differential Diagnosis of Very-Late-Onset SchizophreniaLike Psychosis, Dementia with Lewy Bodies and Alzheimer's Type Dementia with Psychosis. Archives of Clinical Neuropsychology 34, 183-199.

Zarit SH, Zarit JM (2012). Mental disorders in older adults: Fundamentals of assessment and treatment. Guilford Press: Guilford, UK. 